\section{Chlorhexidine Does Not Select for Resistance in Staphylococcus aureus Isolates in a Community Setting}

To the Editor-Chlorhexidine gluconate $(\mathrm{CHG})$ is used in decolonization protocols to prevent invasive Staphylococcus aureus disease in hospital and community settings. ${ }^{1,2}$ Earlier reports have described CHG-resistant $S$. aureus in hospitals, yet evidence of this trend in the community is lacking.,4

A cluster-randomized, double-blind controlled trial evaluating the effectiveness of $2 \%$ CHG body cloths (Sage Products) versus control cloths (Comfort Bath [CB] nonmedicated cleaning cloths; Sage Products) in reducing the incidence of skin and soft-tissue infection (SSTI) was performed in a cohort of military recruits. ${ }^{5}$ Subjects used 2 cloths to cleanse their body 3 times per week. Nasal and axillary body sites were screened for $S$. aureus colonization at baseline and every 2 weeks thereafter during 6 weeks of training. Although CHG did not reduce the incidence of SSTI, it did decrease incident S. aureus colonization.

The substudy presented here evaluated the incidence of CHG-resistant $S$. aureus colonization among these recruits after repeated exposure. Among subjects assigned to the $\mathrm{CHG}$ group, those that were persistently colonized (ie, had results positive for $S$. aureus in nares and/or axilla at baseline and at each biweekly visit) and reported using greater than or equal to $50 \%$ of cloths met criteria for evaluation and had each isolate assessed for $\mathrm{CHG}$ resistance. The same criteria were used for selection of subjects assigned to the $\mathrm{CB}$ group. A random subset of recruits were selected from among subjects in the CB group who met the criteria, and the baseline and last positive biweekly isolates were assessed for $\mathrm{CHG}$ resistance.

CHG minimum inhibitory concentration (MIC) was determined using the agar dilution method and incorporated the use of a 3-mm Steers Replicator. ${ }^{6}$ An ATCC \#29213 S. aureus-positive control with a CHG MIC of $2 \mu \mathrm{g} / \mathrm{mL}$ was used to ensure an expected range of MICs.
No Clinical Laboratory Standards Institute MIC breakpoints exist for CHG. Although correlation of MIC with clinical outcome is unclear, several studies suggest that $\mathrm{CHG}$ MICs can be used as reliable indicators of trends toward decreasing biocide susceptibility. ${ }^{7}$ Furthermore, the genes $q a c A / B$ and $s m r$ encode for drug efflux proteins that confer S. aureus resistance to CHG. Correlation of an elevated MIC $(\geq 4 \mu \mathrm{g} / \mathrm{mL})$ with these genes has been demonstrated. ${ }^{8}$ Polymerase chain reaction for $q a c A / B$ and $s m r$ was performed in duplicate (with positive and negative controls in each run) for isolates with increased CHG MIC as well as for a sample of other isolates from the $\mathrm{CHG}$ and $\mathrm{CB}$ groups.

Of the 781 subjects in the CHG group, $43(5.5 \%)$ met the criteria for evaluation; all 160 isolates from these subjects (3-6 isolates per subject) were evaluated for $\mathrm{CHG}$ resistance. Of the 781 subjects in the $\mathrm{CB}$ group, $58(7.4 \%)$ met the criteria for evaluation. A random sample of 20 of these subjects were selected, and 48 isolates from these subjects were analyzed. Of the 160 isolates from the CHG group that were tested, 25 were methicillin-resistant $S$. aureus (MRSA) and 135 were methicillin-susceptible $S$. aureus (MSSA).

Among the $48 \mathrm{~S}$. aureus isolates obtained from 20 subjects in the $\mathrm{CB}$ group, none exhibited CHG MIC greater than or equal to $4 \mu \mathrm{g} / \mathrm{mL}$ (46 isolates [96\%] had an MIC of $2 \mu \mathrm{g} /$ $\mathrm{mL}$, and 2 isolates [4\%] had an MIC of $1 \mu \mathrm{g} / \mathrm{mL}$ ). Of the 10 isolates tested for $q a c A / B$ and $s m r$, only 1 MSSA isolate was positive for $s m r$.

In the CHG group, a total of 6 isolates (3.8\%) exhibited a CHG MIC of $4 \mu \mathrm{g} / \mathrm{mL}$, all of which isolates were MRSA (Table 1). Two of these isolates, from 2 different subjects, were found at baseline. During the intervention, the remaining 4 isolates were again obtained from these same 2 subjects. The prevalence of $\mathrm{CHG}$ resistance was $4.8 \%$ (95\% confidence interval, $0.57 \%-15.81 \% ; 2$ of 43 subjects), and no incident resistance was identified. The results of molecular analysis for $q a c A / B$ and $s m r$ were negative for the 6 isolates with an MIC of $4 \mu \mathrm{g} / \mathrm{mL}$ as well as for an additional sample of 24 isolates from the CHG group.

Among subjects in both the $\mathrm{CHG}$ and $\mathrm{CB}$ groups combined who were found to be colonized in both the nares and axilla

TABLE 1. Chlorhexidine Gluconate (CHG) Susceptibility of Staphylococcus aureus Isolates from the CHG Group

\begin{tabular}{|c|c|c|c|c|c|c|c|c|}
\hline \multirow[b]{2}{*}{ Period } & \multirow[b]{2}{*}{ No. of isolates tested } & \multicolumn{2}{|c|}{$\begin{array}{l}\text { No. of isolates } \\
\text { tested, by site }\end{array}$} & \multicolumn{2}{|c|}{$\begin{array}{l}\text { No. of isolates } \\
\text { tested, by } \\
\text { susceptibility } \\
\text { to methicillin }\end{array}$} & \multicolumn{3}{|c|}{$\begin{array}{c}\text { Proportion (\%) of } \\
\text { CHG-resistant isolates }\end{array}$} \\
\hline & & Nares & Axilla & MSSA & MRSA & MSSA & MRSA & Total \\
\hline Baseline & 53 & 42 & 11 & 45 & 8 & 0 & $2 / 53(3.8)$ & $2(3.8)$ \\
\hline Intervention & 107 & 82 & 25 & 90 & 17 & 0 & $4 / 107(3.7)$ & $4(3.7)$ \\
\hline Total & 160 & 124 & 36 & 135 & 25 & 0 & $6 / 160(3.8)$ & $6(3.8)$ \\
\hline
\end{tabular}

NOTE. A total of 160 isolates from 43 subjects were tested. MIC, minimum inhibitory concentration; MRSA, methicillin-resistant S. aureus; MSSA, methicillin-susceptible $S$. aureus.

${ }^{a}$ CHG resistance was defined as a minimum inhibitory concentration greater than or equal to $4 \mu \mathrm{g} / \mathrm{mL}$. 
at the same visit $(n=39)$, we found general MIC concordance ( 36 of 39 subjects had isolates with the same MIC value, 2 subjects had discordant pairs with MICs of 1 vs 2 $\mu \mathrm{g} / \mathrm{mL}$, and 1 subject had discordant pairs with MICs of 2 vs $4 \mu \mathrm{g} / \mathrm{mL}$ ). The repeated application of CHG to $S$. aureus strains colonizing military recruits did not select for CHG resistance.

Two hospital-based studies found similar results and reported that no CHG-resistant $S$. aureus isolates were identified after 5- and 7-day treatment courses of daily washes. ${ }^{9,10}$ However, Wang et $\mathrm{al}^{3}$ identified an increase in the incidence of CHG-resistant MRSA (MIC $\geq 4 \mu \mathrm{g} / \mathrm{mL}$ ) over a 20 -year period during which a routine in-hospital CHG hand hygiene program was implemented (the percentage of MRSA isolates with resistance to $\mathrm{CHG}$ increased from $1.7 \%$ to $46.7 \%$ ).

Differences in the incidence of CHG-resistant strains in the hospital versus community setting may be a reflection of the $S$. aureus isolates evaluated. In our community setting, we evaluated predominantly MSSA isolates colonizing recruits, whereas Wang et al $^{3}$ described MRSA isolates associated with blood stream infections in inpatients. Additional studies should investigate potential variability in CHG susceptibility among different strains of $S$. aureus (MSSA vs MRSA, pulsed-field type, colonizing vs infecting, and hospital- vs community-acquired strains).

Our study has several limitations. CHG was used 3 times per week, which differs from the daily application more commonly seen in hospital settings. Adherence to CHG cloths was self-reported, and only $25(23 \%)$ of the 107 isolates that colonized recruits after enrollment day were isolated from the axilla and therefore directly exposed to CHG.

We identified few prevalent and no incident CHG-resistant $S$. aureus strains in a military recruit setting despite repeated exposure to CHG over time. These data support the safety of CHG use as part of a community-based decolonization and SSTI-prevention program.

\section{ACKNOWLEDGMENTS}

We thank Laura Edinger and Frida Stock for their assistance in the microbiology laboratory.

Financial support. Support for this work (IDCRP-001) was provided by the Infectious Disease Clinical Research Program (IDCRP), a Department of Defense program executed through the Uniformed Services University of the Health Sciences. This project has been funded in whole or in part with federal funds from the National Institute of Allergy and Infectious Diseases, National Institutes of Health, under interagency agreement Y1-AI-5072. This study was approved by ethical review committees from the Uniformed Services University of the Health Sciences (protocol IDCRP-001/HU87F7) in compliance with all federal regulations governing the protection of human subjects.

Potential conflicts of interest. All authors report no conflicts of interest relevant to this article. All authors submitted the ICMJE Form for Disclosure of Potential Conflicts of Interest, and the conflicts that the editors consider relevant to this article are disclosed here.
Mark D. Johnson, MD, MTM\&H; ${ }^{1}$

Carey D. Schlett, $\mathrm{MPH}^{3}{ }^{3}$ Greg A. Grandits, $\mathrm{MS}^{4}{ }^{4}$ Katrin Mende, PhD; ${ }^{3,5}$ Timothy J. Whitman, $\mathrm{DO}^{2}$

David R. Tribble, MD, DrPH; ${ }^{3}$

Duane R. Hospenthal, MD, $\mathrm{PhD}{ }^{5}$

Patrick R. Murray, $\mathbf{P h D}^{6}$

Affiliations: 1. Division of Infectious Diseases, Naval Medical Center, San Diego, California; 2. Division of Infectious Diseases, Walter Reed National Military Medical Center, Bethesda, Maryland; 3. Infectious Disease Clinical Research Program, Uniformed Services University of the Health Sciences, Bethesda, Maryland; 4. Division of Biostatistics, University of Minnesota, Minneapolis, Minnesota; 5. Infectious Disease Service, Brooke Army Medical Center, Fort Sam Houston, Texas; 6. National Institutes of Health, Bethesda, Maryland.

Address correspondence to Timothy J. Whitman, DO, Infectious Diseases Department, Walter Reed National Military Medical Center, 8901 Wisconsin Avenue, Bethesda, MD 20889 (timothy.whitman@med.navy.mil).

The views expressed in this article are those of the authors and do not necessarily reflect the official policy or position of the Department of the Navy, Department of the Army, Uniformed Services University of the Health Sciences, Department of Defense, nor the U.S. Government. This work was prepared as part of my official duties. Title 17 U.S.C. 101 defines a U.S. Government work as a work prepared by a military service member or employee of the U.S. Government as part of that person's official duties. National Naval Medical Center PAO reviewed May 2009.

Presented in part: 47th Annual Meeting of the Infectious Diseases Society of America; Philadelphia, Pennsylvania; October 2009.

Infect Control Hosp Epidemiol 2012;33(10):1061-1063

(C) 2012 by The Society for Healthcare Epidemiology of America. All rights reserved. 0899-823X/2012/3310-0019\$15.00. DOI: 10.1086/667744

\section{REFERENCES}

1. Gorwitz RJ, Jernigan DB, Powers JH, Jernigan JA; Participants in the Centers for Disease Control and Prevention Convened Experts' Meeting on Management of MRSA in the Community. Strategies for Clinical Management of MRSA in the Community: Summary of an Experts' Meeting Convened by the Centers for Disease Control and Prevention. Atlanta: Centers for Disease Control and Prevention, 2006.

2. Milstone AM, Passaretti CL, Perl TM. Chlorhexidine: expanding the armamentarium for infection control and prevention. Clin Infect Dis 2008;46(2):274-281.

3. Wang JT, Sheng WH, Wang JL, et al. Longitudinal analysis of chlorhexidine susceptibilities of nosocomial methicillin-resistant Staphylococcus aureus isolates at a teaching hospital in Taiwan. $J$ Antimicrob Chemother 2008;62(3):514-517.

4. Longtin J, Seah C, Siebert K, et al. Distribution of antisepticresistance genes $q a c A / B$ and $s m r$ in methicillin-resistant Staphylococcus aureus isolated in Toronto, 2005-2009. Antimicrob Agents Chemother 2011;2999-3001.

5. Whitman TJ, Herlihy RK, Schlett CD, et al. Chlorhexidineimpregnated cloths to prevent skin and soft-tissue infection in Marine recruits: a cluster-randomized, double-blind, controlled effectiveness trial. Infect Control Hosp Epidemiol 2010;31(12): 1207-1215.

6. Clinical and Laboratory Standards Institute (CLSI). Methods for Dilution: Antimicrobial Susceptibility Test for Bacteria that Grow Aerobically: Approved Standard; Seventh Edition. Wayne, PA: CLSI; 2006. CLSI document M07-A7. 
7. Cookson BD, Bolton MC, Platt JH. Chlorhexidine resistance in methicillin-resistant Staphylococcus aureus or just an elevated MIC? an in vitro and in vivo assessment. Antimicrob Agents Chemother 1991;35(10):1997-2002.

8. Noguchi N, Hase M, Kitta M, Sasatsu M, Deguchi K, Kono M. Antiseptic susceptibility and distribution of antiseptic-resistance genes in methicillin-resistant Staphylococcus aureus. FEMS Microbiol Lett 1999;172(2):247-253.

9. Ridenour G, Lampen R, Federspiel J, Kritchevsky S, Wong E, Climo M. Selective use of intranasal mupirocin and chlorhexidine bathing and the incidence of methicillin-resistant Staphylococcus aureus colonization and infection among intensive care unit patients. Infect Control Hosp Epidemiol 2007;28(10): 1155-1161.

10. Harbarth S, Dharan S, Liassine N, Herrault P, Auckenthaler R, Pittet D. Randomized, placebo-controlled, double-blind trial to evaluate the efficacy of mupirocin for eradicating carriage of methicillin-resistant Staphylococcus aureus. Antimicrob Agents Chemother 1999;43(6):1412-1416.

\section{Retrospective Analysis of Culture-Positive Peripherally Inserted Central Catheter Infections at an Academic Medical Center}

To the Editor-Long-term intravenous (IV) access is often necessary for the administration of medication in the form of antibiotics, total parental nutrition (TPN), and chemotherapy. The trend in long-term IV access has been shifting toward the use of peripherally inserted central catheters (PICCs) instead of surgically placed catheters. PICCs are inserted without the use of general anesthesia and with a lower risk of hemothorax or pneumothorax. Although the convenience of insertion is an advantage, published studies have described various complications such as infection, phlebitis, thrombosis, catheter fracture, and catheter malposition. The intent of this retrospective pilot study was to identify potential modifiable risks associated with culture-documented PICC infections.

The current literature reports no difference in infection rates between central venous catheters and PICCs in hospitalized patients. However, the average life of a PICC in the study was only 11.3 days. ${ }^{1}$ The quoted infection rate for PICCs in all types of patients is reported as approximately $7 \%{ }^{2}$

The Ohio State University (OSU) Wexner Medical Center (OSUWMC) is a 980-bed tertiary-care center in Columbus, Ohio. OSUWMC includes the James Cancer Hospital, an active transplantation program, and a level-1 trauma center. The center has an average of 45,000 admissions annually. The OSUWMC PICC team comprises specifically trained registered nurses (RNs) who are board-certified in vascular access.

A query of the OSUWMC Information Warehouse (IW) database was performed to identify positive culture results for samples collected from all intravenous catheters, including
PICCs, during the period July 1,2008 , to June 30,2009 . The cases identified in this search were then screened to evaluate whether the infection was a true PICC infection. Infection was defined as (1) a positive PICC blood culture result and a negative peripheral blood culture result; (2) a time differential between a positive PICC blood culture result and a positive peripheral blood culture result, with a positive PICC blood culture result occurring first; or (3) a positive PICC or unrecorded site blood culture result and a positive catheter tip culture result ( $>15$ colony forming units) ${ }^{3}$ Clinical patient data were collected and descriptively analyzed for possible risk factors. The OSU Office of Responsible Research Practices Institutional Review Board approved this study.

The search of the IW database identified 126 culture results as indicating possible catheter infections. Results for prisoners were excluded. After screening, 20 PICC infections were identified in 18 evaluable patients. Eleven patients were female. Three (15\%) of 20 of the PICCs involved were placed at an outside facility, the RN PICC team at OSUWMC placed 15 PICCs $(75 \%)$, and the interventional radiology team placed $2(10 \%)$. Three $(17 \%)$ of 18 patients had active malignancies (Epstein-Barr virus [EBV]-associated Burkitt's lymphoma, appendiceal carcinoma, and leiomyosarcoma). The patient with EBV-associated Burkitt's lymphoma was also HIV positive.

The average period from PICC placement until time of infection was 50 days (median, 33 days). In 15 (75\%) of the 20 cases we evaluated, patients had been discharged from the hospital with a PICC in place when the infection occurred. A urinary catheter was present in 13 patients $(65 \%)$ during their hospital stay. Four patients $(20 \%)$ were on a ventilator when their infection occurred, and 4 PICC infections occurred in 3 patients (19\%) who were receiving active chemotherapeutic agents. In addition, 2 other infected patients (10\%), 1 who had multiple sclerosis and 1 who had Castleman disease, were treated with immunosuppressive agents including cyclophosphamide, prednisone, intravenous immunoglobulin, rituxan, and methylprednisolone. Eight $(40 \%)$ of the 20 infections occurred in patients who were receiving TPN. The most common bacterial species isolated was coagulase-negative Staphylococcus species, followed by other gram-positive organisms: Enterococcus species and Staphylococcus aureus.

During a 1-year retrospective review, 20 cases of culturepositive PICC infection were identified at a large academic medical center. In this limited data set, the majority of patients who had PICC infections either had a PICC but were no longer in the hospital or had a urinary catheter in place when their infection developed. About one-third of infections occurred in patients receiving TPN; surprisingly, this is a lower rate than observed in those who were out of the hospital or who had a urinary catheter. TPN has historically been identified as a risk factor for PICC-related infections. In a study by Marra et $\mathrm{al}^{4}$ of 47 patients receiving long-term TPN, $38(80.9 \%)$ of patients developed a catheter-related blood- 